Ansatzpunkte für Kooperationen in der Region Bergisches Land

\section{Ökologische Werkstoffentwicklung im Team}

\section{Seit zwei Jahren existiert in der Bergischen Region ein Netzwerk von Unter- nehmen und Institutionen mit dem Namen K.I.W.I. (1). Eines der gemeinsamen Handlungsfelder ist die "ökologische Werkstoffentwicklung", bei der es u.a. um das Recycling thermoplastischer Kunststoffe der Autozulieferer geht. Eine der zentralen Fragen der Zukunft ist dabei, ob die integrative Problemstellung einer ökologischen Werkstoffentwicklung zu neven Akteurskonstellationen führt, die quer zu den eingefahrenen Beziehungsmustern liegen.}

A Von Rainer Lucas ufgrund der Rahmensetzung durch das Kreislaufwirtschaftsgesetz und die freiwillige Vereinbarung zur Altautoverordnung konzentrieren sich die Bemühungen einer ökologischen Werkstoffentwicklung in der Automobilindustrie derzeit auf Fragen der Wiederverwertung des wachsenden Anteils von Kunststoffteilen (2). Eine wesentliche Voraussetzung, um dem werkstofflichen und rohstofflichen Recycling im Automobilbau einen höheren Stellenwert einzuräumen, ist die Trennung der verschiedenen Kunststofftypen. Die Möglichkeit der Trennung ist wiederum nur gegeben, wenn zum einen die Autoteile werkstofflich gekennzeichnet werden und zum anderen auf den Einsatz von Verbundwerkstoffen bzw. Mischkunststoffen mit Additiven wie Schwermetallen verzichtet wird. „Im Innovationsfeld Recycling sind vor allem im vorgelagerten Bereich der Produktplanung wesentliche Innovationsbedarfe festzustellen. Insgesamt handelt es sich dabei um eine Querschnittsfunktion, die bereits mit der Planung, Konstruktion und Beschaffung beginnt, sich mit der Montage fortsetzt und bis zum Handel als Sammelstelle ausrangierter Teile reicht" (3). Hinsichtlich der externen Unternehmenskontakte ergeben sich aus Sicht der Autoindustrie neue Kooperationsnotwendigkeiten sowohl mit den Vorlieferanten (Autozulieferindustrie) als auch mit benachbarten Branchen (Chemieindustrie als Vorlieferant der Rohstoffe, Kunststoffindustrie, Recyclingwirtschaft).

Um die Frage des Kooperationsinteresses für die in der Region ansässigen Autozulieferfirmen näher zu fassen, ist es notwendig, den TopDown-Blick aus der Sicht der großen Autokonzerne zu verlassen (4). Die Gestaltungs- und Handlungsorientierung in kooperativen Struktu- ren muß sich ja nicht zwangsläufig auf die bestehenden Wertschöpfungsketten beschränken, zumal die große Abhängigkeit vieler Zulieferer von einem Großkunden als problematisch einzustufen ist. Sucht man nach Kooperationsalternativen im Rahmen verschiedener Strategieoptionen der Autozulieferer, so bieten sich zwei Möglichkeiten an:

- Zum einen eine Strategie, die darauf zielt, die Kooperation innerhalb der Branche und bezogen auf das Produkt Auto zu verstärken. Aufgrund der starken Stellung der Automobilhersteller in der Wertschöpfungskette und den neuen Produktionskonzepten sind derartige Kooperationen derzeit einem starken Wandel unterworfen. Die Autoindustrie will die Anzahl der Zulieferfirmen, mit denen sie direkten Kontakt hat, drastisch verkleinern und in der Regel nur noch mit sog. Systemlieferanten kooperieren, die für einzelne Fahrzeugfunktionen Systemlösungen nach zertifizierten Standards anbieten können. Kooperationen zur Anpassung an derartige Vorgaben fördern derzeit die Landesregierungen von NRW und Hessen mit ihren Programmen VIA und MOBIL (5).

- Zum anderen eine Strategie, die die Innovationsvorhaben nicht nur in den bereits existierenden Produktlinien ansiedelt, sondern den gesamten Bereich werkstofflicher Technologien und auch neue Anwendungsfelder umfaßt. Eine solche Option könnte schrittweise dazu führen, daß die Bindungen und Abhängigkeiten im Rahmen bestehender Wertschöpfungspartnerschaften gelockert werden. Für die Region als Ganzes würde diese Kooperationsstrategie auch die Möglichkeit eröffnen, sich von einer Autozulieferregion zur Werkstoffkompetenzregion zu entwickeln.

\section{Rückgriff auf die endogenen} Potentiale der Region?

Für eine ökologische Innovationsstrategie stellt sich die Frage, wie die Firmen überhaupt ökologische Neuerungen und Produktionsalternativen kennenlernen können, die im Rahmen ihrer Produktions- und Werkstofffähigkeiten von Bedeutung sind? Wer regt den Prozeß der Ideenfindung, der am Beginn jeder innovativen Lösung steht, überhaupt an? Welche Rolle können hierbei die regionalen Einrichtungen der Wirtschafts- und Technologieförderungen spielen? Wie können Beratungsleistungen organisiert werden, die für die klein- und mittelständischen Betriebe auch finanzierbar sind? Wer forciert diese Strategien, die eher auf eine Bündelung des unternehmensspezifischen knowhows unter Rückgriff auf die endogenen, regionalen Wissenspotentiale im Bereich des Produktdesigns, der Werkstoffentwicklung und der Oberflächentechnik hinauslaufen?

Die Fragestellungen signalisieren eine Reihe bisher ungelöster Managementprobleme im Rahmen einer regionalen Strukturpolitik in ökologischer Absicht. Ein regionales Netzwerk - wie die K.I.W.I.-Initiative (Das Kürzel steht für Kompetenz, Ideen, Wissenschaft und Innovation) - zwischen Unternehmen, wissenschaftlichen Einrichtungen und den Verwaltungen der Gebietskörperschaften wird hierzu nur etwas beisteuern können, wenn in analytischer, gestalterischer und kommunikativer Hinsicht neue „Policy-Tools“ jenseits des tradierten, ressortmäßigen Verwaltungshandelns entwickelt werden. Notwendig ist zum einen, die vielfältigen Schnittstellenprobleme kommunikativer und inhaltlicher Art mit Bearbeitungskapazitäten auszustatten. Hierfür auch Unternehmen zu gewinnen, die den kooperativen Prozess als Zukunfts-investition behandeln und auch bereit sind, Zeit und damit Geld zu investieren, stellt für den Beginn derartiger Kooperationen eine ernstzunehmende Hürde dar. Zum anderen können regionale Innovationsnetzwerke ihre Aufgaben nur erfuillen, wenn ein praxisbezogener Instrumenten-Mix aus einzelbetrieblichen Technologie- und Organisationsberatungen und weichen, kommunikationsorientierten und kooperativen Verfahren etabliert wird, die sich gegenseitig befruchten. Einen Erfahrungsaustausch zwischen den Technologiezentren der Region, den wirtschaftsnahen Dienstleistern und den Akteuren einer 
regionalisierten Strukturpolitik zu organisieren, ist derzeit in der regionalen Wirtschafts- und Strukturpolitik dringend geboten.

\section{- Projektmanagement statt Regionalmanagement}

Angesichts fehlender institutioneller Kapazitäten werden die angedeuteten Aufgaben eines Kooperationsmanagements derzeit über beteiligungsorientierte Forschungsprojekte zu bewältigen versucht (6). Viele der in der bergischen Region ansässigen klein- und mittelständischen Betriebe der Autozulieferbranche sind mit dem Aufbau von zweiten und dritten Standbeinen aufgrund ihrer geringen Forschungs- und Entwicklungskapazitäten überfordert. Insofern ist $\mathrm{zu}$ klären, wie die regionale Kooperation zwischen Unternehmen, Einrichtungen der Wirtschaftsförderung und der Wissenschaft, sowohl als regelmäßige oder auch nur als nur projektbezogene Beziehung, gestaltet und welche Transfer- und Beratungsangebote im Rahmen der regionalen Netzwerkbildung organisiert werden sollten.

Nach jetzigem Erkenntnisstand (das Projekt arbeitet seit ca. einem halben Jahr) kann es aus Sicht der Unternehmen je nach Anlaß und Aufgabe unterschiedliche Formen der Kooperation geben, z.B.

- als ein Zusammenschluß mit anderen kleinen Firmen der gleichen Branche in der Region, die mit ähnlichen Problemstellungen konfrontiert sind (Kooperation in der traditionellen Wertschöpfungskette),

- als eine Kooperation zwischen kunststoffverarbeitenden Autozulieferfirmen, der Recyclingwirtschaft und der chemischen Industrie entlang spezieller Stoffströme (Kooperation in neuen Wertschöpfungsketten),

- als eine Kooperation mit regionalen Forschungseinrichtungen und Technologiezentren (Kooperation als wechselseitiger Austausch von Informationen).

Alle drei Optionen setzen die Bereitschaft bei den Unternehmen voraus, den betrieblichen Handlungsrahmen zu öffnen und Zeit und Ressourcen in einen Kooperationsprozess einzubringen. Fine aktuelle Studie zu Kooperationen in der Kfz-Zulieferindustrie (7) stellt hierzu fest, daß im Bereich Entwicklung und Innovation die höchsten Erfolgspotentiale gesehen werden. Produktion, Materialwirtschaft und Logistik folgen mit vergleichbaren Gewichtungen. Betriebe, die unmittelbaren Problemdruck verspüren, werden u.U. eher zu Kooperationen bereit sein; allerdings ist der Erwartungsdruck an die Kooperation dann besonders groß, was wiederum zu Frustationen führen kann, wenn sich kein unmittelbarer Erfolg einstellt. Damit keine falschen Erwartungen geweckt werden, sollte die Zielsetzung der Kooperation am Anfang klar umrissen werden.

Kooperationsmöglichkeiten im Recyclingverbund

Für die kunststoffverarbeitenden Betriebe der Autozulieferindustrie bestehen im Rahmen eines möglichen Recyclingverbundes - bei dem eine Rïckfuihrung von Altstoffen in den Produktionsprozess erfolgt - zwei grundlegend unterschiedliche Kooperationsalternativen mit unterschiedlichen räumlichen Perspektiven: zum einen ein eher zentralistischer, großräumiger Recyclingverbund im Rahmen der traditionellen produktbezogenen (Automobil)-Wertschöpfungsketten, zum anderen ein eher regionaler Verbund, entlang von materialbezogenen Wertschöpfungsketten zwischen der Recyclingwirtschaft und der kunststoffverarbeitenden Industrie (siehe auch Abbildung 1). Beiden Optionen wird im Rahmen der Forschungsarbeiten am IÖW sowohl hinsichtlich der kooperativen Gestaltungsperspektiven als auch der umweltpolitischen Wirkung nachgegangen werden.

\section{Bedingungen für erfolgreiche Kooperationen}

Die Handlungsfelder für regionale Kooperationen sind von Region zu Region unterschiedlich und sicher auch durch vielfältige Faktoren begrenzt. Trotzdem lassen sich einige Elemente insbesondere in den Beziehungen der Unternehmen zu den regionalen Transfereinrichtungen verallgemeinern. Die Möglichkeiten regions-spezifischer Transferleistungen basieren auf gewachsenen Strukturen und bereits vorhandenen Netzwerken, in denen bereits eine langjährige Erfahrung zur Lösung der Probleme in den Unternehmen vorhanden ist. Gerade dieses Erfahrungswissen aufgrund unmittelbarer Kontakte zu Betrieben und deren Managern macht regionale Lösungen insbesondere für klein- und mittelständische Unternehmen attraktiv. Nicht abstraktes Wissen ist gefragt, sondern anwendungsbezogene Hilfen. Dies setzt Personen und Einrichtungen voraus, die leicht erreichbar sind und denen man vertrauen kann (auch hinsichtlich der preislichen Gestaltung ihrer Leistungen).

Die Bedingungen regionaler Kooperation können aufgrund der bisherigen Erfahrungen in der K.I.W.I.-Initiative wie folgt systematisiert werden:

\section{Abbildung 1: Kooperationsalternativen beim Recycling von Kunststoffteilen}

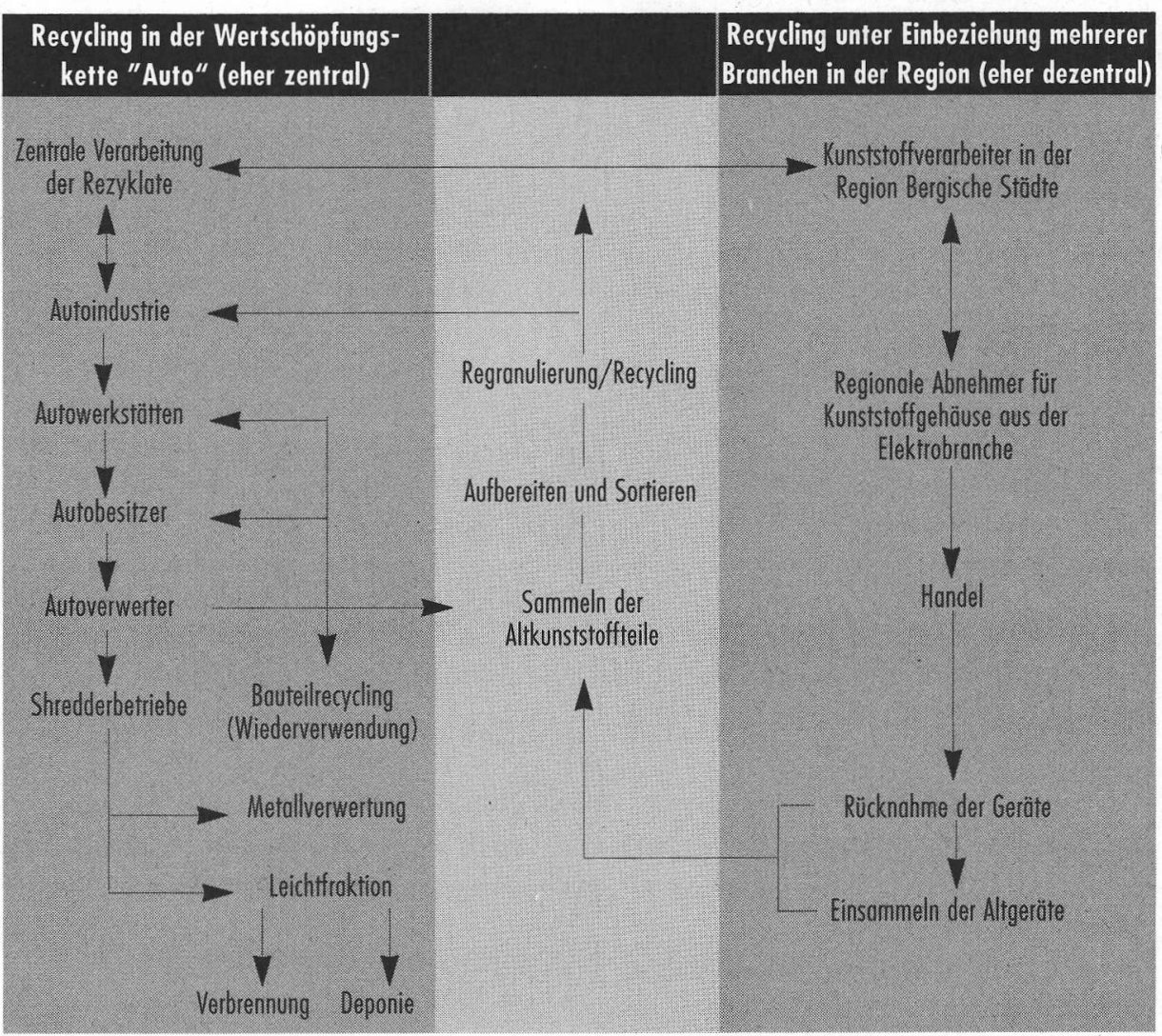


- Eine Verständigung auf eine gemeinsame, ökologische und ökonomische Zielperspektive sollte am Anfang stehen. Der Übergang von der problemzentrierten Kooperation zur strategischen Kooperation gelingt nur, wenn gemeinsame Ziele definiert werden, deren Umsetzung dann durchaus in pragmatischen Teilschritten erfolgen kann.

- Das unternehmenseitige Interesse hat viele Facetten: Handlungsdruck, Unzufriedenheit mit bestehenden Partnerschaften, Aufbau neuer Geschäftsfelder. Ein Unternehmensmix aus großen und kleinen, ökologisch fortgeschrittenen und weniger fortgeschrittenen sollte unter dem Gesichtspunkt „Unternehmen lernen von Unternehmen " zunächst in kleinen, überschaubaren Arbeitskreisen angeboten werden.

- Für partnerschaftliche Strategien (Verbundlösungen) sind darüber hinaus vertrauensbildende Maßnahmen besonders wichtig (z.B. positiver persönlicher Eindruck von möglichen Partnern, gegenseitiger Besuch in den Betriebsstätten);

- Die ökologische Werkstoffentwicklung ist eine strategische Frage und sollte damit Angelegenheit der Unternehmensfïhrung sein. Erst die Verbindung von Recycling, Produktnutzen und Marketing schafft die notwendige Basis für die Realisierung eines Nutzens.

- Ein leichter Zugang zu bestehenden Informationen und Transferangeboten; transparente Beratungsangebote nicht nur $\mathrm{zu}$ technischen
Fragen ist eine wichtige Voraussetzung für die Attraktivität eines regionalen Netzwerkes. Die Informationsbarrieren und sonstigen Zugangsprobleme zu Personen und Institutionen müssen gegenüber überregionalen Angeboten deutlich geringer sein. Zur Verminderung der Transaktionskosten bedarf es daher einer angebotsorientierten Strategie der Transferstellen und Beratungseinrichtungen.

Ein derartiger Prozess steht in der Region Bergische Städte noch am Anfang. Er hat mit der K.I.W.I.-Initiative einen Impulsgeber und Vermittler. Wenn dieser Prozess jedoch nicht nur durch einzelne Projekte und damit befristet begleitet werden soll, ist es notwendig, das K.I.W.I.-Management mit dauerhaften, nicht projektgebundenen personellen und materiellen Ressourcen auszustatten bzw. eine Transferstelle einzurichten, die derartige Vermittlungsaufgaben übernimmt. Denn regionale Kooperation ist immer mit zusätzlichen Integrationsleistungen (fachlich und personell) verbunden. Insofern sind zumindest in der Anfangsphase personelle und finanzielle Mittel notwendig, um in ökologischen Handlungsfeldern zu neuen und tragfähigen Kooperationsformen zu kommen.

\section{Literafur}

1) Lucas, R./ Weißner, B. (1997): Entwicklung unternehmerischer Innovationspotentiale im Rahmen der K.I.W.I. Initiative der Region Bergisches Städredreieck, IÖW-Schriftenreihe, im Erscheinen
2) Schlotter, U. (1996), Werkstoffliche Verwertung von Kunststoffen aus Alffahrzeugen, in: Kunststoffe 8/96, S.1084-1087, München sowie Schmidt, J./Leithner, R. (Hg.) (1995): Automobil-Recycling, Berlin/Heidelberg/New York

3) Lompe, K.; Blöcker, A.; Lux, B.; Syring, 0. (1996): Regionalisierung als Innovationsstrategie; Berlin, S. 130 4) vgl. hierzu auch: Region Bergisches Städtedreieck Remscheid-Solingen-Wuppertal (1993):

Automobilzulieferer und Strukturwandel, Zukunft der Automobilzulieferindustrie im Bergischen Land

5) Verbundinitiative Automobil NRW (1995): Der Automobilstandort Nordrhein-Westfalen; Stand November 1995; Hessisches Ministerium für Wirtschaft, Verkehr, Technologie und Europaangelegenheiten (1993): MOBIL - Verbundinitiative "Hessische Automobilzulieferer"; Wiesbaden

6) Eine Selbstdarstellung des Projektes "Ökologische Werkstoffkomptenz, betriebliche Innovation und regionale Kooperation", das von der Deutschen Bundesstiffung Umwelt finanziert wird, kann über das IÖW-Regionalbüro in NRW bezogen werden. Ein erster Workshop ist für Ende Mai geplant.

7) vgl. Diebold Deutschland GmbH (1996): Kooperationen in der Kfz-Zulieferindustrie - Umfrage-Ergebnisse, Eschborn

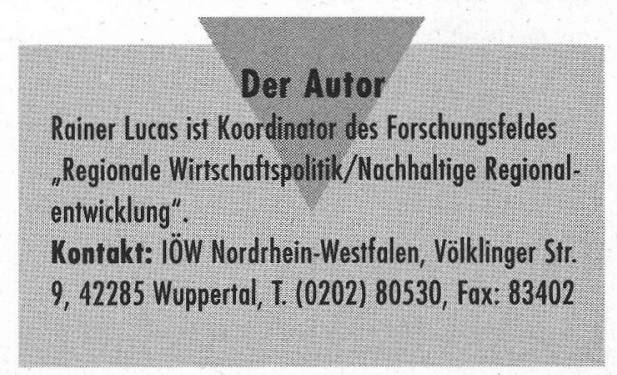

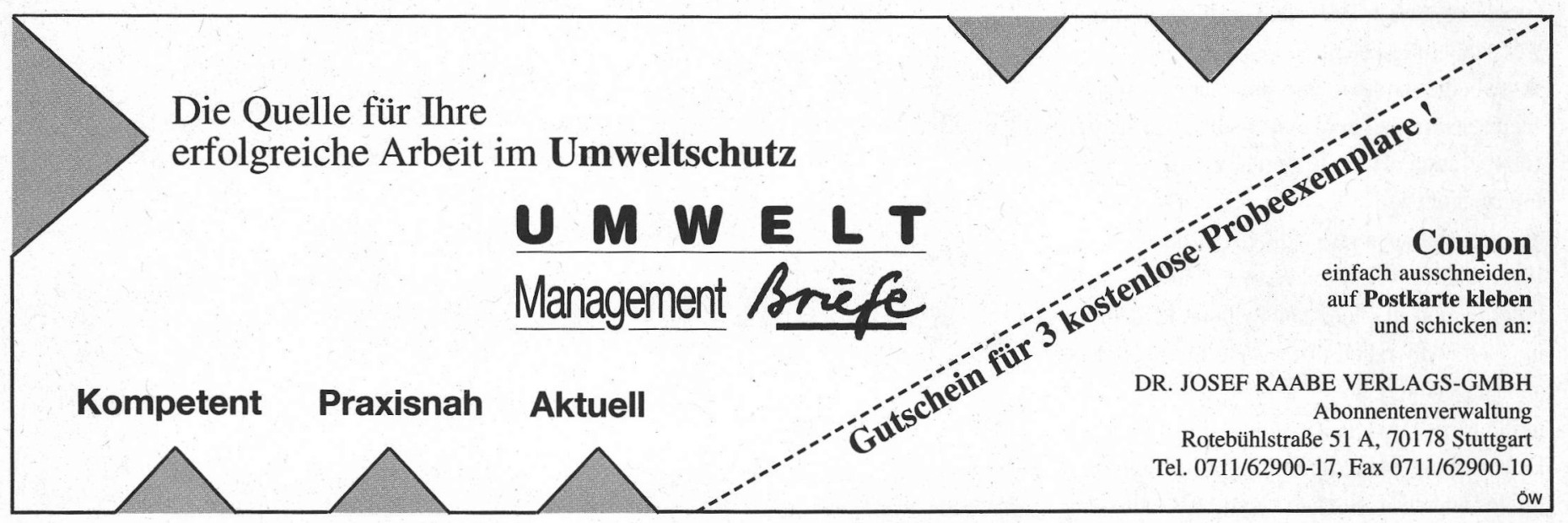


(c) 20I0 Authors; licensee IÖW and oekom verlag. This is an article distributed under the terms of the Creative Commons Attribution Non-Commercial No Derivates License (http://creativecommons.org/licenses/by-nc-nd/3.o/), which permits unrestricted use, distribution, and reproduction in any medium, provided the original work is properly cited. 